\title{
Effect of Non-Uniform Temperature Gradients on the Onset of Convection in a Couple-Stress Fluid-Saturated Porous Medium
}

\author{
I. S. Shivakumara ${ }^{1 \dagger}$, S. Sureshkumar ${ }^{2}$ and N. Devaraju ${ }^{1}$ \\ ${ }^{1} U G C$-Centre for Advanced Studies in Fluid Mechanics \\ Department of Mathematics, Bangalore University, Bangalore-560001, India \\ ${ }^{2}$ Department of Mathematics, Siddaganga Institute of Technology, Tumkur-572103, India \\ $\dagger$ Corresponding Author Email: iskumar2006@gmail.com
}

(Received January 16, 2010; accepted April 5, 2010)

\begin{abstract}
The effect of different forms of basic temperature gradients on the criterion for the onset of convection in a layer of an incompressible couple-stress fluid-saturated porous medium is investigated. It is shown that the principle of exchange of stability is valid, and the eigenvalue problem is solved numerically using the Galerkin technique. The parabolic and inverted parabolic basic temperature profiles have the same effect on the onset of convection and similar is the case between piecewise linear temperature profiles heating from below and above. Amongst the various basic temperature profiles, the linear temperature profile is found to be more stabilizing on the onset of instability. In addition, the influence of thermal depth on the criterion for the onset of convection is assessed in the case of piecewise linear temperature profiles. Moreover, an increase in the value of couple-stress parameter is found to delay the onset of convection and to increase the width of convection cells. It is also noted that the critical wave numbers are slightly affected by the nature of basic temperature profiles.
\end{abstract}

Keywords: Couple-stress fluid, Porous medium, Convection, Non-uniform temperature gradient.

\section{NOMENCLATURE}

$\begin{array}{ll}a & \text { overall horizontal wave number } \\ A_{h} & \text { ratio of heat capacities } \\ D & \text { differential operator } \\ f(z) & \text { non-dimensional temperature gradient } \\ K & \text { permeability of the porous medium } \\ \ell, m & \begin{array}{l}\text { horizontal wave numbers in the } x \text { and } y \\ \text { directions respectively }\end{array} \\ M & \text { non-dimensional group } \\ p & \text { pressure } \\ P r_{D} & \text { modified Darcy- Prandtl number } \\ \vec{q} & \text { seepage velocity } \\ R_{D} & \text { Darcy- Rayleigh number } \\ t & \text { time } \\ T & \text { temperature }\end{array}$

\section{INTRODUCTION}

Thermal convective instability in a layer of porous medium has merited extensive attention over the years and is now emerged as an important field of study in the general area of fluid dynamics and heat transfer. The growing volume of work pertaining to this field is well

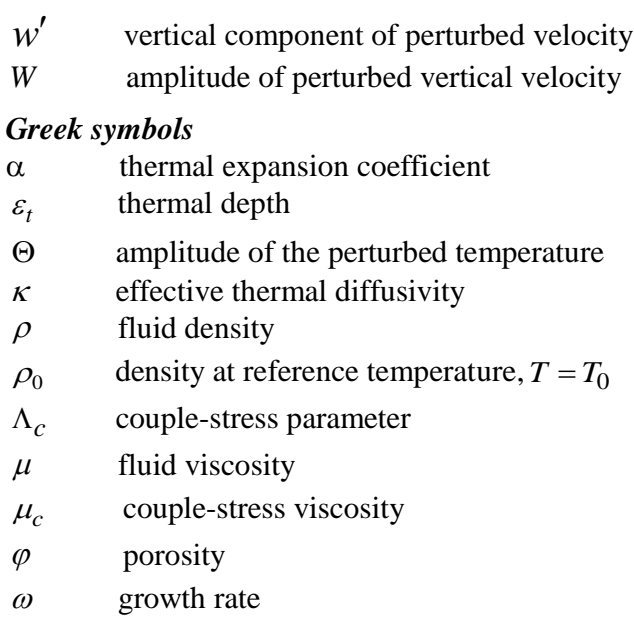

documented by Ingham and Pop (1998), Vafai (2000, 2005) and Nield and Bejan (2006). In many of the heat transfer problems, suppress or augment of convection plays a vital role. There are several mechanisms that can be used effectively to either suppress or augment 
convectCion namely, by applying a magnetic/electric field externally or by Coriolis force due to rotation or by maintaining non-uniform temperature gradient across the porous layer. A non-uniform temperature gradient can arise in various ways, notably by (i) transient heating or cooling at a boundary, (ii) volumetric distribution of heat sources, (iii) radiative heat transfer, (iv) thermal modulation, and (v) vertical throughflow. A large cross section of the fundamental research carried out on these topics has been covered extensively in Nield and Bejan (2006).

Although several studies have been undertaken in the past to understand convective instability using nonuniform basic temperature gradients, all the investigations have been limited to Newtonian fluidsaturated porous media. Nevertheless, the growing importance of non-Newtonian fluids in modern technology has attracted researchers for the consideration of such fluids in the investigation of convective instability problems because the traditional Newtonian fluids cannot precisely describe the characteristics of the fluid flow encountered in many practical problems such as the extrusion of polymer fluids, solidification of liquid crystals, cooling of metallic plates in a bath, exotic lubricants and colloidal fluids to mention a few. These fluids deform and produce a spin field due to the microrotation of suspended particles forming a micropolar fluid. The theory of micropolar fluids was developed by Eringen (1966) which takes care of local effects arising from the microstructure and as well as the intrinsic motions of microfluidics. The spin field due to microrotation of freely suspended particles sets-up an antisymetric stress, known as couple stress, and thus forming couple stress fluid. Thus couple-stress fluid, according to Eringen (1966), is a particular case of micropolar fluid when microrotation balances with the natural vorticity of fluid. The couple-stress fluid has distinct features, such as polar effects and whose microstructure is mechanically significant. The constitutive equations for couple-stress fluids are given by Stokes (1966). The theory proposed by Stokes is the simplest one for micro-fluids, which allows polar effects such as the presence of couple-stress, body couple, and nonsymmetric tensors.

Based on this formulation, convective instability in either a couple-stress fluid layer or in a couple-stress fluid-saturated porous layer heated from below has been investigated in the recent past. Sharma and Thakur (2000) have investigated thermal instability of an electrically conducting couple-stress fluid-saturated porous layer in the presence of a uniform magnetic field. They have reported that the couple-stress delays the onset of stationary convection. A layer of couplestress fluid saturating a porous medium heated from below in the presence of rotation has been studied by Sharma et al. (2000) and condition for the onset of convection is obtained. Sunil et al. (2002) have investigated the effect of magnetic field and rotation on a layer of couple-stress fluid heated from below in a porous medium, while Sunil et al. (2004) have considered the effect of suspended particles in a couplestress fluid layer heated and soluted from below in a porous medium. The linear and non-linear double diffusive convection with Soret effect in couple-stress liquids has been considered by Malashetty et al. (2006). Gaikwad et al. (2007) have studied linear and nonlinear double diffusive convection with Soret and Dufour effects in couple-stress liquids. Recently, Malashetty et al. (2009) have studied convective instability in a layer of couple-stress fluid-saturated porous medium heated from below using a thermal nonequilibrium model.

All these previous investigators have considered the effect of only uniform temperature gradient on the onset of convection in a couple-stress fluid-saturated porous medium. Nevertheless, the effect of non-uniform basic temperature gradients concerning this problem has not been given any attention in the literature despite its importance in understanding convective instability encountered in many scientific and technological problems. The novelty of the present work is to understand which basic temperature profile leads to the least critical Darcy-Rayleigh number. The answer is not trivial and it is not answered even in the case of Newtonian fluids for isothermal boundaries and the available results are restricted to only insulating boundaries since this case is amenable for analytical treatment (Shivakumara 2009). The intent of the present study is, therefore, to analyze the influence of different forms of nonuniform basic temperature profiles, namely (i) linear temperature profile, (ii) parabolic temperature profile, (iii) inverted parabolic temperature profile, (iv) piecewise linear temperature profile heating from below and $(\mathrm{v})$ piecewise linear temperature profile cooling from above and make clear their effects on the onset of convection in a couple-stress fluid-saturated isotropic porous medium for isothermal boundaries.

To achieve the above objectives, the paper is organized as follows. The mathematical formulation is given in section 2 and it is shown that the principle of exchange of stabilities is valid irrespective of the form of basic temperature profile. The method of solution to solve the eigenvalue problem is discussed in section 3. The results are discussed in section 4 and conclusions drawn are presented in section 5 .

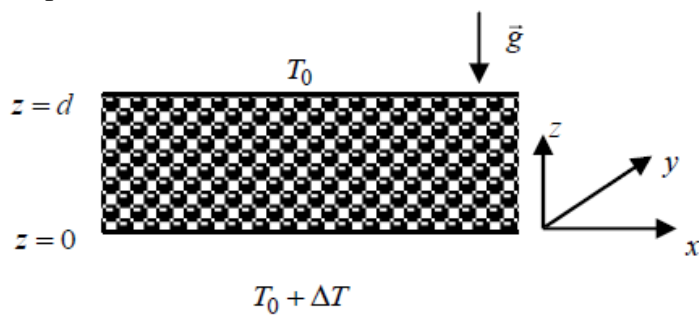

Fig. 1. Physical configuration

\section{MATHEMATICAL FORMUlation}

We consider an infinite horizontal layer of an incompressible couple-stress fluid-saturated porous medium heated from below, where the vertical distance between the top and bottom boundaries is $d$ and a fixed temperature difference $\Delta T$ is maintained across the porous layer(see Fig.1). A Cartesian coordinate system $(x, y, z)$ is used with the origin at the bottom of the porous layer and the z-axis vertically upward in the gravitational field. 
Under the Boussinesq approximation, the equation of continuity and the equation of state are, respectively

$$
\begin{aligned}
& \nabla \cdot \vec{q}=0 \\
& \rho=\rho_{0}\left\{1-\alpha\left(T-T_{0}\right)\right\} .
\end{aligned}
$$

The equation of motion of an incompressible couplestress fluid saturating a porous medium, following Malashetty et al. (2009), is given by

$$
\frac{1}{\varphi} \frac{\partial \vec{q}}{\partial t}=-\frac{1}{\rho_{0}} \nabla p+\frac{\rho}{\rho_{0}} \vec{g}-\frac{1}{\rho_{0} K}\left(\mu-\mu_{c} \nabla^{2}\right) \vec{q}
$$

The time derivative term is taken into consideration in Eq. (3) in order to look into the possibility of oscillatory convection. When $\mu_{c}=0$, Eq. (3) reduces to the usual modified Darcy equation which describes the flow of a Newtonian fluid saturating a porous medium and note that the presence of couple-stress is to alter the viscosity of the fluid.

The temperature equation is given by

$A_{h} \frac{\partial T}{\partial t}+(\vec{q} \cdot \nabla) T=\kappa \nabla^{2} T$

Since the non-uniform basic temperature profile finds its origin in transient heating or cooling at a boundary, the temperature distribution in the basic state usually depends explicitly on position and time. Thus, the quiescent basic state is described by

$\vec{q}_{b}=0, p=p_{b}(z, t), \frac{\partial T_{b}}{\partial t}=\kappa \frac{\partial^{2} T_{b}}{\partial z^{2}}$

where the subscript $b$ denotes the basic state. As propounded by Nield (1975), it is also not our intention to treat here the full problem of temperature profiles depending explicitly on time. Instead, we introduce a simplification in the form of a quasi-static approximation which consists of freezing the basic temperature distribution $T_{b}(z, t)$ at a given instant of time. This simplification is justified so long as the disturbances are growing faster than the basic profile is evolving (Nield and Bejan 2006). Under the circumstances, the basic state temperature distribution admits a solution of the form

$-\frac{d}{\Delta T} \frac{d T_{b}}{d z}=f(z)$

where $f(z)$ is a non-dimensional basic temperature gradient satisfies the condition

$$
\int_{0}^{1} f(z) d z=1 .
$$

To study the stability of the basic state, we superimpose infinitesimally small perturbations $(\stackrel{\mathrm{r}}{q} \phi, p \phi, q \phi, r \phi)$ on the basic state in the form

$$
\vec{q}=\vec{q}^{\prime}, \quad p=p_{b}+p^{\prime}, T=T_{b}+\theta^{\prime}, \rho=\rho_{b}+\rho^{\prime} .
$$

Following the standard linear stability analysis procedure, non-dimensionalizing the quantities by scaling $(x, y, z)$ by $d, t$ by $d^{2} \varphi / \kappa, w^{\prime}$ by $\kappa / d, \theta^{\prime}$ by $\Delta T$, and employing the normal mode analysis procedure in the form

$\left(w^{\prime}, \theta^{\prime}\right)=(W, \Theta)(z) \exp [i(\ell x+m y)+\omega t]$

we obtain the stability equations in the form

$$
\begin{aligned}
& {\left[\frac{\omega}{P r_{D}}+1-\Lambda_{c}\left(D^{2}-a^{2}\right)\right]\left(D^{2}-a^{2}\right) W=-R_{D} a^{2} \Theta} \\
& {\left[M \omega-\left(D^{2}-a^{2}\right)\right] \Theta=f(z) W .}
\end{aligned}
$$

In the above equations, $D=d / d z$ is the differential operator, $a=\sqrt{l^{2}+m^{2}}$ is the overall horizontal wave number, $\Lambda_{c}=\mu_{c} / \mu d^{2}$ is the couple-stress parameter, $R_{D}=\alpha g \Delta T d K / \nu \kappa$ is the Darcy- Rayleigh number, $\operatorname{Pr}_{D}=v \varphi^{2} d^{2} / K \kappa$ is the modified Darcy- Prandtl number, and $M=A_{h} / \varphi$ is a non-dimensional group.

The boundaries of the porous layer are assumed to be impermeable with vanishing couple-stress and perfect conductors of heat. Accordingly, the boundary conditions are

$W=D^{2} W=\Theta=0 \quad$ at $z=0,1$.

To prove the validity of principle of exchange of stability, the moment approach is used which does not suffer from the ambiguities of satisfying some of the higher boundary conditions (Khalili et al. 2002). To derive the moment equation, we multiply Eq. (9) by $W^{m}$ and Eq. (10) by $\Theta^{m}$, where $m$ is a non-negative integer, and then integrate from $z=0$ to 1 . Many terms are integrated by parts and simplified using the boundary conditions, to get

$$
\begin{gathered}
\left(\frac{\omega}{P r_{D}}+1\right)<D W^{m} D W+a^{2} W^{m} W>+\Lambda_{c}<D^{2} W^{m} D^{2} W> \\
+<-2 a^{2} D W^{m} D W+a^{4} W^{m} W>=R_{D} a^{2}<W^{m} \Theta> \\
\left(M \omega+a^{2}\right)<\Theta^{m} \Theta>+<D \Theta^{m} D \Theta>=<f(z) \Theta^{m} W>
\end{gathered}
$$

Here $m=0$ gives the moment equation and $m=1$ gives the energy equation. For $m=0$, Eq. (12) and Eq. (13), respectively reduce to

$$
\begin{aligned}
& {\left[\frac{\omega}{P r_{D}}+1+\Lambda_{c} a^{2}\right]<W>=R_{D}<\Theta>} \\
& \left(M \omega+a^{2}\right)<\Theta>=<f(z) W>.
\end{aligned}
$$

Eliminating $\langle\Theta>$ from Eq. (14) by using Eq. (15) and rearranging the terms, we obtain

$$
\begin{gathered}
\frac{M}{P r_{D}} \omega^{2}+\left\{M\left(1+\Lambda_{c} a^{2}\right)+\frac{a^{2}}{P r_{D}}\right\} \omega+\left(1+\Lambda_{c} a^{2}\right) a^{2} \\
-R_{D} \frac{\langle f(z) W>}{<W>}=0
\end{gathered}
$$


Since we are interested in the occurrence of oscillatory convection, we set $\omega=i \omega_{i}$, where $\omega_{i}$ is real, in Eq. (16) and obtain

$$
\begin{array}{r}
\frac{M}{P r_{D}} \omega_{i}^{2}-i\left\{M\left(1+\Lambda_{c} a^{2}\right)+\frac{a^{2}}{P r_{D}}\right\} \omega_{i}-\left(1+\Lambda_{c} a^{2}\right) a^{2} \\
+R_{D} \frac{<f(z) W>}{<W>}=0
\end{array}
$$

Equating the real and imaginary parts of Eq. (17), we, respectively, note that

$\frac{M}{P r_{D}} \omega_{i}^{2}-\left(1+\Lambda_{c} a^{2}\right) a^{2}+R_{D} \frac{<f(z) W>}{<W>}=0$

and

$\left\{M\left(1+\Lambda_{c} a^{2}\right)+\frac{a^{2}}{P r_{D}}\right\} \omega_{i}=0$.

Equation (19) implies that $\omega_{i}=0$, and hence, the principle of exchange of stability is valid. Therefore, we confine the analysis to stationary convection and take $\omega=0$ in Eqs. (9) and (10) to arrive at the following system of ordinary differential equations:

$$
\left[\Lambda_{c}\left(D^{2}-a^{2}\right)-1\right]\left(D^{2}-a^{2}\right) W=R_{D} a^{2} \Theta
$$

$\left(D^{2}-a^{2}\right) \Theta=-f(z) W$.

The following five different forms of $f(z)$ are considered to investigate their effect on the criterion for the onset of convection:

(i) Linear temperature profile

$$
f(z)=1 \text {. }
$$

(ii) Piecewise linear temperature profile heating from below

$$
f(z)=\left\{\begin{array}{ll}
\frac{1}{\varepsilon_{t}}, & 0 \leq z<\varepsilon_{t} \\
0, & \varepsilon_{t}<z \leq 1
\end{array} .\right.
$$

(iii) Piecewise linear temperature profile cooling from above

$$
f(z)= \begin{cases}0, & 0 \leq z<1-\varepsilon_{\mathrm{t}} \\ \frac{1}{\varepsilon_{\mathrm{t}}}, & 1-\varepsilon_{\mathrm{t}}<z \leq 1\end{cases}
$$

(iv) Inverted parabolic temperature profile

$$
f(z)=2(1-z) \text {. }
$$

(v) Parabolic temperature profile

$$
f(z)=2 z \text {. }
$$

\section{Method OF Solution}

Equations (20) and (21) together with boundary conditions (11) constitute an eigenvalue problem with $R_{D}$ as the eigenvalue. The resulting eigenvalue problem is solved using the Galerkin technique.
Accordingly, the unknown variables are written in a series of basis functions as

$W=\sum_{i=1}^{N} A_{i} W_{i}, \Theta=\sum_{i=1}^{N} B_{i} \Theta_{i}$

where $A_{i}$ and $B_{i}$ are constants and the basis functions $W_{i}$ and $\Theta_{i}$ will be represented by the power series satisfying the respective boundary conditions. Substituting Eq. (27) into Eqs. (20) and (21), multiplying the resulting momentum equation by $W_{j}(z)$, energy equation by $\Theta_{j}(z)$, performing the integration by parts with respect to $\mathrm{z}$ between $\mathrm{z}=0$ and $\mathrm{z}=1$ and using the boundary conditions (11), we obtain the following system of linear homogeneous algebraic equations:

$C_{j i} A_{i}+D_{j i} B_{i}=0$
$E_{j i} A_{i}+F_{j i} B_{i}=0$.

The coefficients $C_{j i}$ to $F_{j i}$ involve inner products of the base functions and are given by

$$
\begin{aligned}
& C_{j i}=\Lambda_{c}<D^{2} W_{j} D^{2} W_{i}+a^{4} W_{j} W_{i}+2 a^{2} D W_{j} D W_{i}>+<D W_{j} D W_{i}+a^{2} W_{j} W_{i}> \\
& D_{j i}=-R_{D} a^{2}<W_{j} \Theta_{i}> \\
& E_{j i}=<f(z) \Theta_{j} W_{i}> \\
& F_{j i}=-<D \Theta_{j} D \Theta_{i}+a^{2} \Theta_{j} \Theta_{i}>
\end{aligned}
$$

where the inner product is defined as $\langle\cdots\rangle=\int_{0}^{1}(\cdots) d z$.

The above set of homogeneous algebraic equations given by Eq. (29) will have a non-trivial solution if and only if

$\left|\begin{array}{cc}C_{j i} & D_{j i} \\ E_{j i} & F_{j i}\end{array}\right|=0$.

We select trial functions satisfying the appropriate boundary conditions. The following polynomial trial functions are chosen:

$$
\begin{aligned}
& W_{i}=\left(z^{i+1}-2 z^{i+2}+z^{i+3}\right) T_{i-1}^{*} \\
& \Theta_{i}=\left(z^{i}-z^{i+1}\right) T_{i-1}^{*}
\end{aligned}
$$

where $T_{i}^{*}{ }^{\prime} s$ are the modified Chebyshev polynomials. The inner products involved in Eq. (30) are evaluated analytically rather than numerically to avoid errors in the numerical integration. For a fixed value of $\Lambda_{c}, \varepsilon_{t}$ and for chosen basic temperature profile $f(z)$, Eq. (30) gives a relation between $R_{D}$ and $a$ which enables us to plot a locus in the $\left(R_{D}, a\right)$ - plane. The DarcyRayleigh numbers for basic temperature profiles (i) to (v) are respectively denoted through $R_{D 1}$ to $R_{D 5}$. The minimum point of $R_{D}$ as a function of $a$ as well as with respect to $\varepsilon_{t}$ (in the case of piecewise linear 
temperature profiles), gives the critical Darcy-Rayleigh number $R_{\text {Dic }}$ ( $i=1$ to 5 ) and the corresponding critical wave number $a_{i c}$, as well as critical thermal depth depending on the choice of basic temperature profile.

\section{RESULTS AND DISCUSSION}

The effect of different forms of basic temperature gradients on the criterion for the onset of convection in a layer of couple-stress fluid-saturated porous medium is investigated. The resulting eigenvalue problem is solved numerically using the Galerkin technique. The convergence of the results is achieved, in general, by considering six terms in the series expansion Eq. (27) and the results obtained for this order (i.e., $N=6$ ) are presented graphically in Figs. 2 to 5. However, for a linear temperature profile (i.e., $f(z)=1$ ) the resulting eigenvalue problem can be solved exactly with the eigen functions $W(z)=A_{1} \sin \pi z$ and $\Theta(z)=$ $A_{2} \sin \pi z$, where $A_{1}$ and $A_{2}$ are constants. In this case, an analytic expression for the Rayleigh number, denoted by $R_{D 1}$, is obtained in the form

$R_{D 1}=\frac{\left(\pi^{2}+a^{2}\right)^{2}\left\{\Lambda_{c}\left(\pi^{2}+a^{2}\right)+1\right\}}{a^{2}}$.

It is noted that $R_{D 1}$ attains its critical value, $R_{D 1 c}$ at $a^{2}=a_{1 c}^{2}$, where

$a_{1 c}^{2}=\frac{-\left(\pi^{2} \Lambda_{c}+1\right)+\sqrt{\left(\pi^{2} \Lambda_{c}+1\right)\left(9 \pi^{2} \Lambda_{c}+1\right)}}{4 \Lambda_{c}}$.

When $\Lambda_{c}=0$, the critical Rayleigh number and the critical wave number are respectively found to be $R_{D 1 c}=4 \pi^{2}$ and $a_{1 c}=\pi$ which are the known exact values. From the above equations, it is also evident that an increase in the value of couple-stress parameter $\Lambda_{c}$ is to increase the Rayleigh number and to decrease the critical wave number. In fact, $a_{1 c} \rightarrow \pi / \sqrt{2}$ as $\Lambda_{c} \rightarrow \infty$.

Figures $2 \mathrm{a}$ and $2 \mathrm{~b}$ depict the neutral stability curves in the $\left(R_{D i}, a\right)$ - plane for different temperature profiles for $\Lambda_{c}=0$ and 1 , respectively with $\varepsilon_{t}=0.5$. The coordinates of the minimum point on these curves correspond to the critical values $R_{D i c}$ and $a_{i c}$ ( $i=1$ to $5)$. From these figures, it can be seen that the critical wave number is slightly affected by the nature of the basic temperature profiles. The numerical calculations of Darcy-Rayleigh numbers for different values of wave number revealed that $R_{D 2}=R_{D 3}$ as well as $R_{D 4}=R_{D 5}$

. That is, parabolic and inverted parabolic temperature profiles have the same effect on the onset of convection and similar is the case between piecewise linear temperature profiles heating from below and cooling from above. This may be due to the symmetric boundary conditions considered. Furthermore, increasing $\Lambda_{c}$ increases the critical Rayleigh number and thus the presence of couple-stresses is to delay the onset of convection.
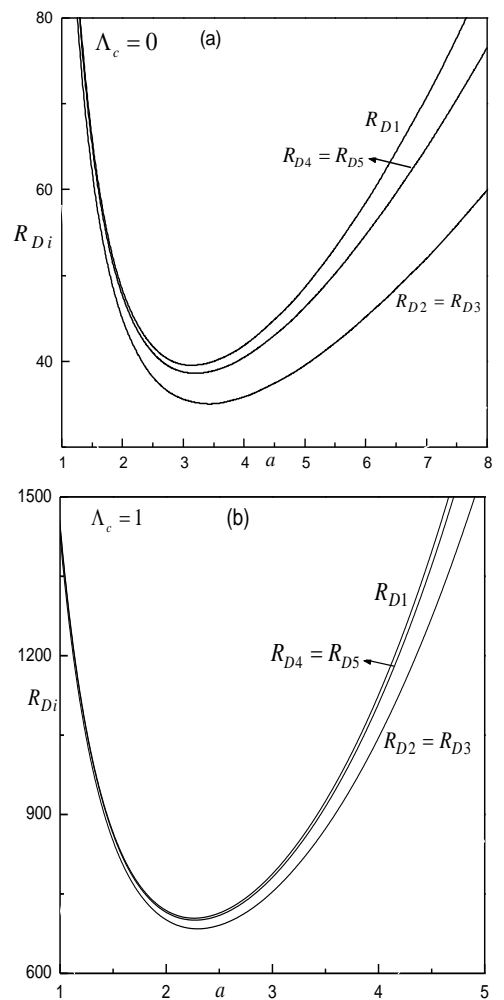

Fig. 2. Neutral stability curves for different temperature profiles

The critical Darcy-Rayleigh numbers obtained with respect to the wave number for three values of $\Lambda_{c}(=0$, 0.1 and 0.2 ) are represented graphically in Fig. 3 as a function of thermal depth $\varepsilon_{t}$ for piecewise linear temperature profiles. From the figure it is seen that the critical Darcy-Rayleigh numbers decrease at first to some minimum value and then increase steadily with further increase in $\varepsilon_{t}$. The critical value of $\varepsilon_{t}$ is found to be 0.71 and it is independent of $\Lambda_{c}$ values considered. Also, increasing $\Lambda_{c}$ increases the critical Darcy-Rayleigh number and hence its effect is to reinforce stability of the system.

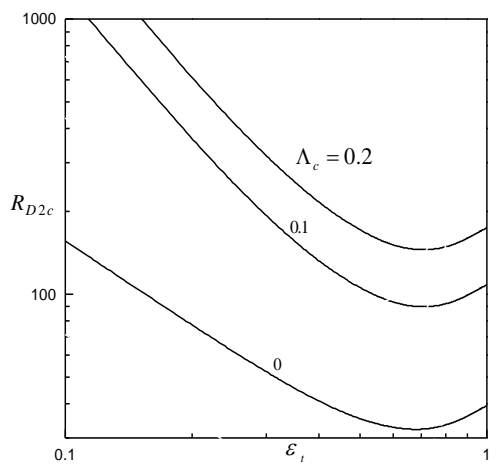

Fig. 3. Variation of $R_{D 2 c}$ with $\varepsilon_{t}$ for different values of $\Lambda_{c}$ 


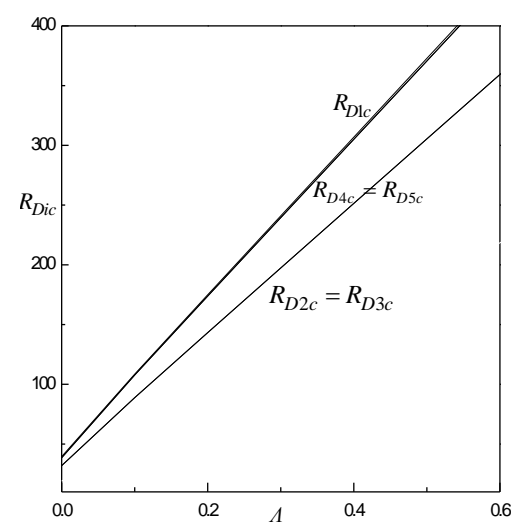

Fig. 4. Variation of $R_{D i c}(i=1$ to 5$)$ as a function of $\Lambda_{c}$

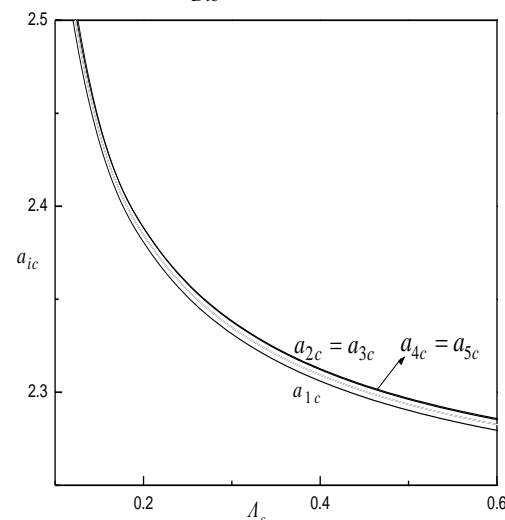

Fig. 5. Variation of $a_{i c} \quad(i=1$ to 5$)$ as a function of $\Lambda_{c}$

Figures 4 and 5, respectively show the variation of critical Darcy-Rayleigh numbers ( $R_{\text {Dic }}, i=1$ to 5 ) and the corresponding critical wave numbers ( $a_{i c}, i=1$ to 5 ) as a function of $\Lambda_{c}$. The critical Darcy-Rayleigh numbers presented here are obtained with respect to the wave number as well as $\varepsilon_{t}$ (in the case of piecewise linear temperature profiles). From Figure 4, it is observed that the critical Darcy-Rayleigh numbers increase monotonically with $\Lambda_{c}$, and it is further noted that

$$
R_{D 2 c}=R_{D 3 c}<R_{D 4 c}=R_{D 5 c}<R_{D 1 c} .
$$

This indicates that, as compared to non-uniform temperature profiles, the linear temperature profile has a more stabilizing effect on the system. In particular, it may be noted that the system is more unstable for piecewise linear temperature profiles as compared to parabolic/inverted parabolic basic temperature profiles. However, the difference in the critical Darcy-Rayleigh numbers for different basic temperature profiles is seen to be marginal.

The critical wave numbers for different temperature profiles decrease monotonically with an increase in $\Lambda_{c}$ as may be seen from the results plotted in Fig. 5. Thus an increase in $\Lambda_{c}$ is seen to increase the size of convection cells. Further inspection of the figure reveals that the deviation in the critical wave numbers among different temperature profiles is not so significant and in fact $a_{2 c}\left(=a_{3 c}\right)$ values are almost same as $a_{4 c}\left(=a_{5 c}\right)$.

\section{Conclusions}

From the foregoing discussions it can be concluded that (i) the effect of increasing the couple-stress parameter $\Lambda_{c}$ is to stabilize the onset of instability and also (ii) the difference between critical values at each $\Lambda_{c}$ for the various chosen basic temperature profiles is minimal. In this sense, there is very little influence that one can assert on the instability of the system by the choice of various basic temperature profiles different from linear. Therefore, one can simply use the analytic solution for the linear temperature profile to assess the strong effect of couple-stress parameter $\Lambda_{c}$ on the onset of convection in a couple-stress fluid saturated porous medium. In addition, the parabolic or inverted parabolic temperature profiles and also piecewise linear temperature profiles heating from below or cooling from above have the same effect on the onset of convection in a couple-stress fluid-saturated porous medium. This may be attributed to symmetric boundary conditions. The critical thermal depth $\varepsilon_{t c}$ is found to be same for piecewise linear temperature profiles and remains invariant with change in $\Lambda_{c}$ values. The critical wave numbers are slightly affected by the nature of basic temperature profiles. Moreover, an increase in the value of $\Lambda_{c}$ is to enlarge the size of convection cells.

\section{ACKNOWLEDGEMENTS}

This work was supported by the UGC-Centre for Advanced Studies in Fluid Mechanics. One of the authors (SSK) would like to thank the Director, Principal and Management of Siddaganga Institute of Technology, Tumkur for the encouragement. The authors wish to thank the reviewer for useful comments which have lead to the improvement of the paper.

\section{REFERENCES}

Eringen, A.C. (1966). Theory of micropolar fluids. $J$. Math. Mech. 16, 1-18.

Gaikwad, S.N., M.S. Malashetty and K. Ramaprasad (2007). An analytical study of linear and nonlinear double diffusive convection with Soret and Dufour effects in couple stress fluid. Int. J. Nonlinear Mech. 42(7), 903-813.

Ingham, D.B. and I. Pop. (1998). Transport Phenomena in Porous Media. Pergamon, Oxford.

Khalili, A., I.S. Shivakumara and M. Huettel (2002). Effects of throughflow and internal heat generation on convective instabilities in an anisotropic porous layer. J. of Porous Media 5, 187-198.

Malashetty, M.S., I.S. Shivakumara and K. Sridhar (2009). The onset of convection in a couple stress fluid saturated porous layer using a thermal nonequilibrium model, Physics Letters A 373, 781790.

Malashetty, M.S., S.N. Gaikwad and Mahantesh Swamy (2006). An analytical study of linear and 
I.S. Shivakumara et al. / JAFM, Vol. 5, No. 1, pp. 49-55, 2012.

non-linear double diffusive convection with Soret effect in couple stress liquids. Int. J. Thermal sciences 45(9), 897-907.

Nield, D.A. and A. Bejan (2006). Convection in Porous Media. (3rd ed.) Springer- Verlag, New York.

Sharma, R.C. and K.D. Thakur (2000). On couple-stress fluid heated from below in porous medium in hydromagnetics. Czechoslovvak J. Physics 50(6), 753-758.

Sharma, R.C., Sunil and Mohinder Pal (2000). On couple-stress fluid heated from below in porous medium in presence of rotation. Appl. Mechanics and Engg. 5(4), 883-896.

Shivakumara, I.S. (2010). Onset of convection in a couple-stress fluid-saturated porous medium: effects of non-uniform temperature gradients. Arch. Appl. Mech. 80, 949-957.

Stokes, V. K. (1966). Couple stresses in fluids. Phys. of Fluids 91 (1966) 1709-1715.

Sunil, R.C., Sharma and Mohinder Pal (2002). On couple-stress fluid heated from below in porous medium in presence of magnetic field and rotation. J. of Porous Media 5(2), 149-158.

Sunil, R.C., Sharma and Rajender Singh Chandel (2004). Effect of suspended particles on couplestress fluid heated and soluted from below in porous medium. J. Porous Media 7(1), 9-18.

Vafai, K. (2000). Handbook of Porous Media. Marcel Dekker, New York.

Vafai, K. (2005). Handbook of Porous Media. Taylor and Francis/CRC, Boca Raton, FL. 